\title{
Bilayer Thickness Mismatch Controls Domain Size in Model Membranes
}

\author{
Frederick A. Heberle, ${ }^{*}{ }^{\dagger}$ Robin S. Petruzielo, ${ }^{\S}$ Jianjun Pan, ${ }^{\dagger}$ Paul Drazba, ${ }^{\perp}$ Norbert Kučerka, $\nabla, \square$ \\ Robert F. Standaert, ${ }^{\dagger, \dagger, \# ~ G e r a l d ~ W . ~ F e i g e n s o n, ~}$, and John Katsaras $*, \dagger, \perp, \nabla, \diamond$ \\ ${ }^{\dagger}$ Biology \& Soft Matter and ${ }^{\star}$ Biosciences Divisions, Oak Ridge National Laboratory, Oak Ridge, Tennessee 37831, United States \\ ${ }^{\S}$ Departments of Physics and "Molecular Biology and Genetics, Cornell University, Ithaca, New York 14853, United States \\ ${ }^{\perp}$ Departments of Physics and ${ }^{\#}$ Biochemistry and Molecular \& Cellular Biology, University of Tennessee, Knoxville, Tennessee 37996, \\ United States

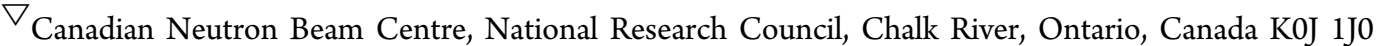 \\ $\square$ Department of Physical Chemistry of Drugs, Faculty of Pharmacy, Comenius University, 83232 Bratislava, Slovakia

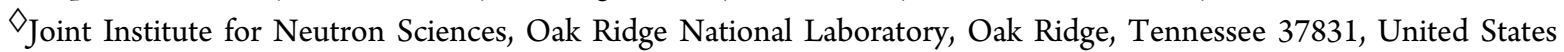

Supporting Information

\begin{abstract}
The observation of lateral phase separation in lipid bilayers has received considerable attention, especially in connection to lipid raft phenomena in cells. It is widely accepted that rafts play a central role in cellular processes, notably signal transduction. While micrometer-sized domains are observed with some model membrane mixtures, rafts much
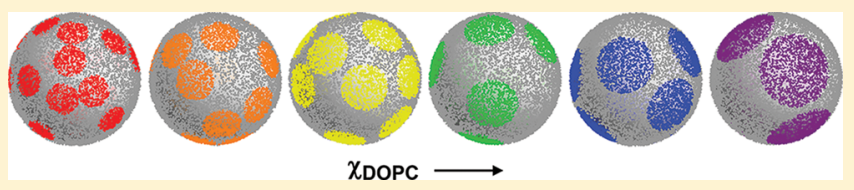
smaller than $100 \mathrm{~nm}$ - beyond the reach of optical microscopy-are now thought to exist, both in vitro and in vivo. We have used small-angle neutron scattering, a probe free technique, to measure the size of nanoscopic membrane domains in unilamellar vesicles with unprecedented accuracy. These experiments were performed using a four-component model system containing fixed proportions of cholesterol and the saturated phospholipid 1,2-distearoyl-sn-glycero-3-phosphocholine (DSPC), mixed with varying amounts of the unsaturated phospholipids 1-palmitoyl-2-oleoyl-sn-glycero-3-phosphocholine (POPC) and 1,2-dioleoylsn-glycero-3-phosphocholine (DOPC). We find that liquid domain size increases with the extent of acyl chain unsaturation (DOPC:POPC ratio). Furthermore, we find a direct correlation between domain size and the mismatch in bilayer thickness of the coexisting liquid-ordered and liquid-disordered phases, suggesting a dominant role for line tension in controlling domain size. While this result is expected from line tension theories, we provide the first experimental verification in free-floating bilayers. Importantly, we also find that changes in bilayer thickness, which accompany changes in the degree of lipid chain unsaturation, are entirely confined to the disordered phase. Together, these results suggest how the size of functional domains in homeothermic cells may be regulated through changes in lipid composition.
\end{abstract}

\section{INTRODUCTION}

The spatial organization of lipids and proteins in biological membranes seems to have a functional role in the life of a cell. Diverse experimental evidence supports the participation of small lipid domains (rafts) in membrane processes including protein sorting, vesicular transport, viral entry and exit from cells, and signaling. Raft functionality may well involve the reversible coalescence and growth of intrinsically small and transient domains into larger structures that act as platforms for organizing protein machinery. ${ }^{1}$ Despite intense interest, the fundamental mechanisms controlling domain size transitions have remained elusive.

Theoretical work has sought to establish a link between membrane domain size and the different structural and mechanical properties of liquid-disordered (Ld) and liquidordered (Lo) phases (reviewed in ref 2). Of central importance is the boundary energy at the domain/surround interface, expressed as a line tension, which arises in part from the presumed thickness mismatch between the domain and its surround, and concomitant unfavorable exposure of the membrane's hydrocarbon region to water. Various mechanisms allow the bilayer to minimize this exposure, including elastic deformations at the boundary ${ }^{3}$ and reduction of the boundary perimeter through domain coalescence. ${ }^{4}$ Domain size and lifetime distributions result from the interplay between these and other (perhaps unknown) mechanisms. Importantly, the data necessary to properly evaluate theoretical work and point toward new directions are lacking, in part because crucial parameters, including nanometer-scale bilayer thicknesses and domain sizes, have proven difficult to measure in free-floating vesicular bilayers.

A less well-recognized but equally significant barrier to experimental progress has been a lack of detailed knowledge of the compositions of coexisting phases, which precludes systematic study of the differential properties predicted to

Received: November 19, 2012

Published: February 7, 2013 

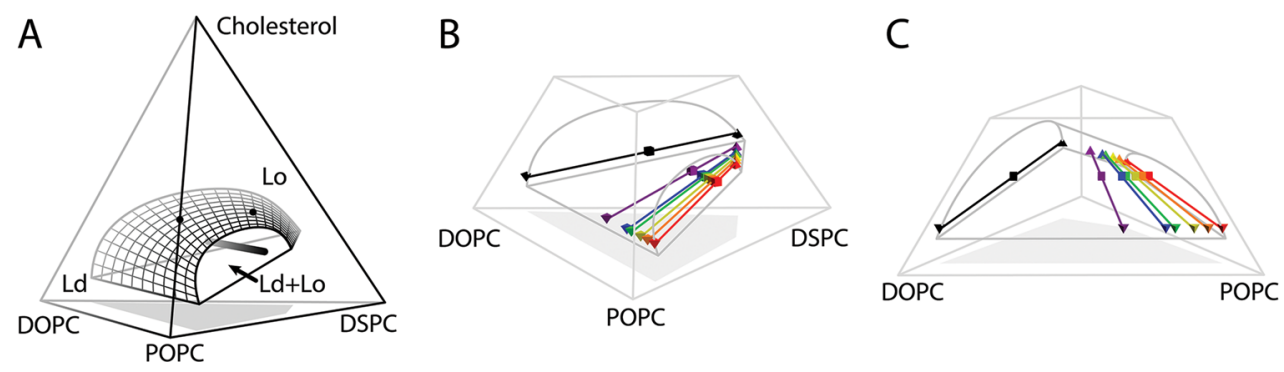

Figure 1. Phase behavior of samples examined in this study. (A) The four-component phase diagram for DSPC/DOPC/POPC/Chol at $20{ }^{\circ} \mathrm{C}$ is represented as a tetrahedron, where shading indicates the relative amount of POPC (black) and DOPC (light gray). The domain sample trajectory (solid line) is contained within the Ld+Lo region (shown bounded by mesh lines). Single phase control samples S1 and S2 (black circles) lie on the DSPC/POPC/Chol three-component face of the tetrahedron, and the binary POPC/Chol edge, respectively. (B and C) two views of the Ld+Lo region showing the sample compositions (cubes) and their corresponding Ld (down-pointing pyramids) and Lo (up-pointing pyramids) tieline end point compositions: D1 (red), D2 (orange), D3 (yellow), D4 (green), D5 (blue), D6 (purple), and D7 (black).

affect domain organization. Substantial progress has now been made in this regard, resulting in many complete and partial phase diagrams for biologically relevant lipid mixtures (reviewed in refs 5 and 6). Building on this foundation, we recently characterized a domain size transition, from nanometers to micrometers, in the four-component lipid mixture 1,2distearoyl-sn-glycero-3-phosphocholine (DSPC), 1,2-dioleoyl$s n$-glycero-3-phosphocholine (DOPC), 1-palmitoyl-2-oleoyl-snglycero-3-phosphocholine (POPC) and cholesterol (Chol), ${ }^{7}$ for which the detailed phase behavior had been previously established. $^{8,9}$ Remarkably, instead of a gradual change in domain size, three distinct regimes of spatial organization were observed in the liquid phase coexistence region, controlled by the relative amounts of lipids with different degree of acyl chain unsaturation. (i) When the low-melting lipid fraction was composed predominantly of DOPC, micrometer-sized domains were observed in giant unilamellar vesicles (GUVs) with fluorescence microscopy. (ii) At high fractions of POPC, GUVs appeared uniform, but a heterogeneous lipid distribution was revealed by fluorescence resonance energy transfer (FRET) and electron spin resonance (ESR); together, these observations imply the presence of nanoscopic domains. (iii) Between these composition extremes, a narrow transition zone appeared, characterized by spatially modulated domain patterns. In this transition zone, striking changes in domain size and morphology were effected by relatively minor changes in the structure of the low-melting lipid, implying a key role for mixture composition in controlling raft phenomena in cell plasma membranes.

Of these three regimes of domain size and shape, the least understood is the regime of nanoscopic phases, which is inaccessible to most biophysical techniques, including fluorescence microscopy. Here, we use small-angle neutron scattering (SANS) to measure the composition dependence of membrane domain size in pure lipid systems free from extrinsic probes, with unprecedented nanometer accuracy. We find a correlation between domain size and bilayer thickness differences in the coexisting Ld and Lo phases, also measured with SANS. This is the first experimental study to confirm the theoretically predicted connection between domain size and membrane thickness mismatch in free-floating bilayers. Specifically, we find that the degree of acyl chain unsaturation in the low-melting lipids (i.e., POPC and DOPC) controls the size of nanodomains.

\section{RESULTS}

We examined the temperature and composition dependence of membrane domain formation in the four-component lipid mixture DSPC/DOPC/POPC/Chol. A partial room-temperature phase diagram for this mixture emphasizing the region of coexisting liquid phases $(\mathrm{Ld}+\mathrm{Lo})$ is shown in Figure 1. The compositions studied here are listed in Table S1 (Supporting Information, SI), and lie on a trajectory within the Ld+Lo region. All compositions on this trajectory have fixed mole fractions of DSPC, (DOPC+POPC), and Chol, in a 39/39/22 ratio; the single variable parameter is the percentage of DOPC in the low-melting lipid fraction. For convenience in referring to this key parameter, we define $\rho \equiv \mathrm{DOPC} /(\mathrm{DOPC}+\mathrm{POPC})$ [\%], such that increasing values of $\rho$ represent an increasing degree of acyl chain unsaturation in the bilayer. Our study focuses on the regime of nanoscopic $(0<\rho<15 \%)$ and modulated $(15<\rho<25 \%)$ phases previously observed in GUVs. $^{7}$

SANS Detects Domain Formation in Model Membranes. We used small angle neutron scattering (SANS) to observe domain formation in $60 \mathrm{~nm}$ diameter unilamellar vesicles (ULVs), as shown schematically in Figure 2. Small angle scattering results from nonuniform scattering length

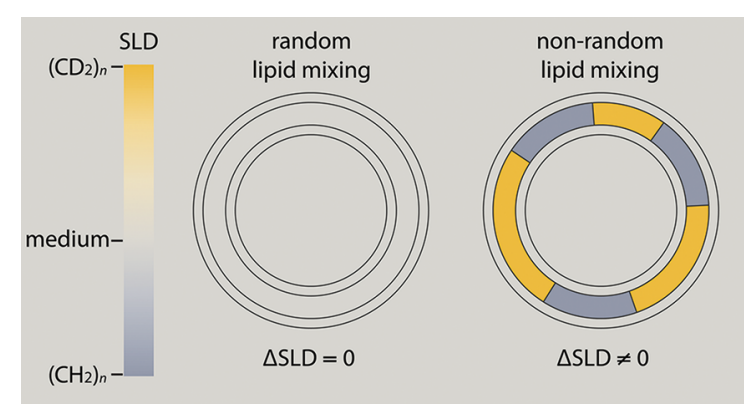

Figure 2. Schematic of a SANS contrast matching experiment. Lipid phase separation is driven by immiscibility of saturated (e.g., stearoyl) and unsaturated (e.g., oleoyl) acyl chains. Domain detection requires a scattering length density (SLD) contrast between the coexisting phases. This contrast is achieved by using chain-perdeuterated DSPC, which has a significantly higher SLD than protiated POPC or DOPC. Left, the SLD of the aqueous medium is matched to that of the acyl chain and headgroup regions in the ULV (shown in cross section), such that random mixing of lipids within the bilayer plane results in a null contrast condition. Right, segregation of saturated and unsaturated chains results in lateral SLD fluctuations, and a scattering signal. 
density (SLD) in the sample volume. For ULVs, SANS arises from three components: ${ }^{10}$ (i) a mean component from SLD contrast between the average bilayer composition and the solvent; (ii) a radial component from SLD variation in the direction normal to the bilayer plane (we note that the radial SLD profile of a lipid bilayer can be approximated by two slabs corresponding to the headgroup and acyl chain regions); and (iii) a lateral component from SLD variation within the plane of the bilayer. The SLD of the solvent and acyl chains can be controlled experimentally by adjusting the ratio of $\mathrm{D}_{2} \mathrm{O} / \mathrm{H}_{2} \mathrm{O}$ in the aqueous medium, and the ratio of deuterated/protiated chains in the bilayer. We matched the average SLD of the solvent, headgroup, and acyl chain region (Tables S1 and S2, SI) using a $34.6 \% \quad \mathrm{D}_{2} \mathrm{O}$ medium, and $65.4 \%$ of chainperdeuterated DSPC- $\mathrm{d}_{70}$ (expressed as a percentage of total DSPC). Under these conditions, minimal scattering is observed from the nearly contrast-free system that exists when lipids are randomly mixed within the plane of the bilayer (e.g., at high temperature). Upon domain formation, however, the lateral segregation of saturated and unsaturated species generates contrast in the acyl chain region, resulting in an increased scattering signal.

Figure 3 shows SANS intensity $I$ as a function of scattering vector $\mathrm{q}$ and temperature. Composition $\mathrm{S} 1$ consists of a single liquid phase at $T \geq 20{ }^{\circ} \mathrm{C} .{ }^{9}$ No measurable scattering is observed over the ranges $0.005<q<0.06 \AA^{-1}$ and $20 \leq T \leq 50$ ${ }^{\circ} \mathrm{C}$ for this sample, consistent with a contrast-free system, where the lipids are randomly mixed. Samples D1-D7 also scatter negligibly at $50{ }^{\circ} \mathrm{C}$, but behave differently than $\mathrm{S} 1$ at lower temperatures, where domains form. A scattering peak at low $q\left(0.005-0.02 \AA^{-1}\right)$ emerges as the temperature is reduced to $40{ }^{\circ} \mathrm{C}$, which increases in magnitude upon further lowering of the temperature. At fixed temperature, the magnitude of the peak increases with increasing DOPC fraction (i.e., in the series D1-D7). These trends are quantified in Figure 4, which plots the total integrated scattering intensity $Q=\int I(q) q^{2} \mathrm{~d} q$. As mentioned previously, the total scattered intensity for ULVs can be expressed as the sum of three components, which are related to the square of the mean SLD contrast (i.e., the difference between the bilayer and water SLDs), the mean square radial SLD fluctuations, and the mean square lateral SLD fluctuations. From comparison of the single-phase samples S1 and S2 to the phase-separated (domain) samples D1-D7, it is evident that lateral contrast, rather than mean or radial contrast (which to first order affects all samples equally), is the dominant source of scattering. (Further discussion of this point is found in Section S1, SI).

Membrane Domain Size Correlates with Ld/Lo Thickness Mismatch. SANS intensity at $20{ }^{\circ} \mathrm{C}$ was modeled by simulating the SLD contrast-weighted pair-distance distribution function $P(r)$, as described in Materials and Methods. Precise tieline information from the phase diagram allowed us to fix the volume fractions and SLDs of coexisting phases, such that the single remaining unknown was the domain size. A simple model describes a vesicle of radius $R$ containing $N$ circular $L d$ domains of radius $R_{\mathrm{d}}$, where the total domain volume fraction is constrained to equal the Ld volume fraction calculated from the phase diagram and published lipid volumes (Section S2.1, SI).

For each mixture composition, we varied the single parameter $N$ to achieve a best fit to the SANS data. Figure 5 shows the fitting results for compositions D1-D6. The low $q$ peak in SANS intensity is related to the average distance between points within a domain (effectively the domain size)
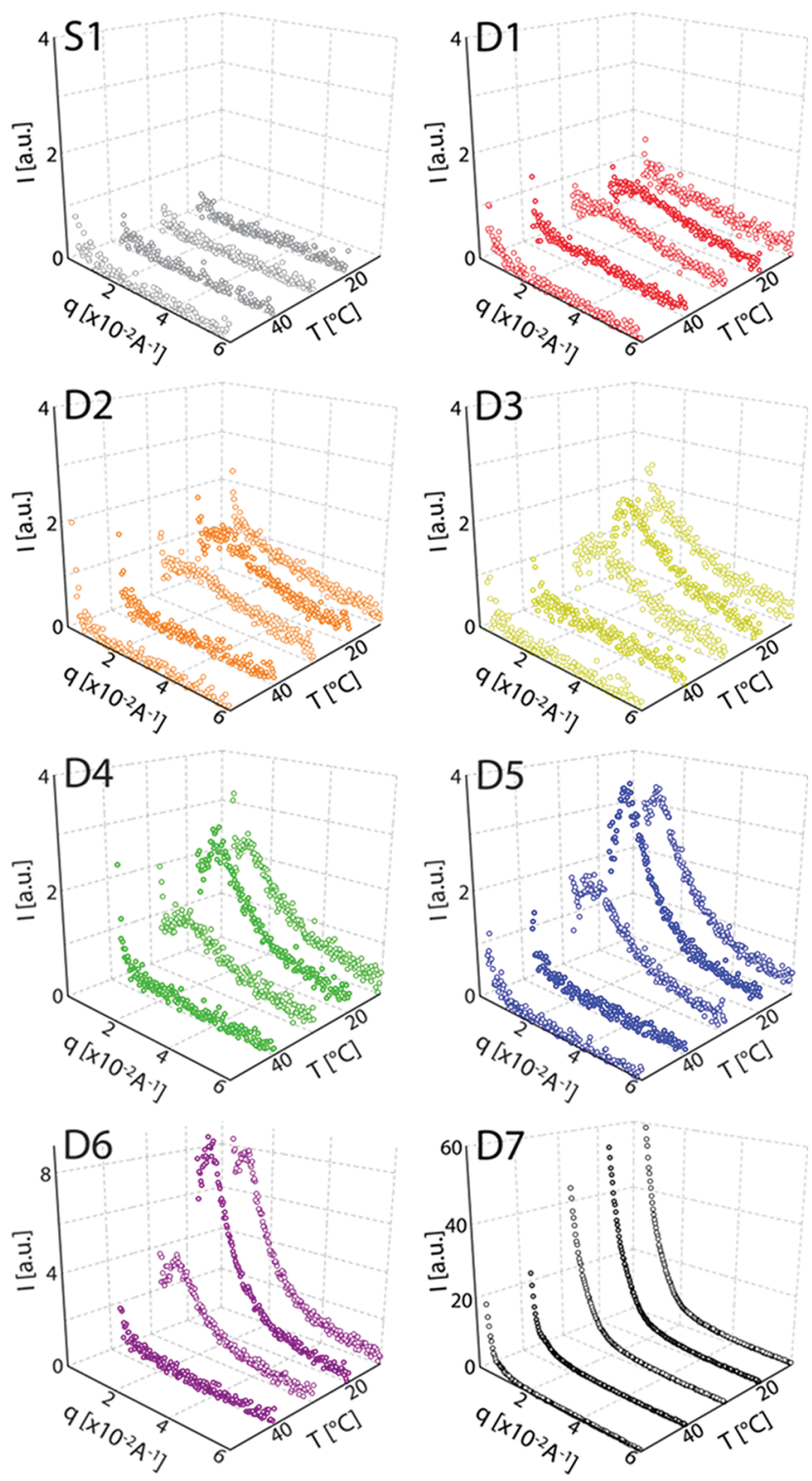

Figure 3. SANS depends on temperature and bilayer composition. Samples D1-D6 are plotted on an expanded scale relative to D7 to facilitate comparison. Sample compositions are given in Table S1 of the SI, and coloring corresponds to Figure 1.

and should therefore shift to lower $q$ with increasing domain size. This trend is observed in the data and quantified by the model, through which $R_{\mathrm{d}}$ can be derived from $N$. Effective domain radius increases from $68 \AA(\sim 250$ lipids per leaflet) at $\rho$ $=0$, to $162 \AA(\sim 1500$ lipids per leaflet $)$ at $\rho=35 \%$. The trend is plotted in Figure 6, revealing a linear dependence of $R_{\mathrm{d}}$ on DOPC fraction. Over the same compositional range, $N$ decreases from 23 to 6 . Together, the changes in domain size and number decrease the total domain perimeter, relative to the perimeter of a single domain of the same area, by a factor of 2 (Table 1). A further reduction in domain perimeter ratio is observed for composition D7 $(\rho=100 \%)$. The $20^{\circ} \mathrm{C}$ scattering curve for D7 does not possess a distinct maximum (an observation consistent with large domains, for which the scattering peak occurs below the experimental $q$ window) and is not well fit by a single value of $N$. However, the data can be fit satisfactorily with a linear combination of $N=1$ and $N=4$ 


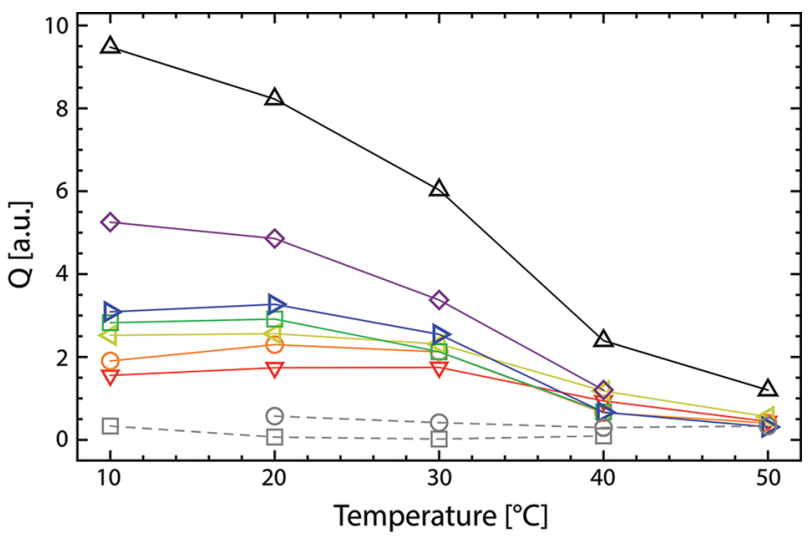

Figure 4. Total scattering reveals the temperature dependence of domain formation for single-phase control samples S1 (gray circle) and S2 (gray square), and domain samples D1 (red down-pointing triangle), D2 (orange circle), D3 (yellow left-pointing triangle), D4 (green square), D5 (blue right-pointing triangle), D6 (purple diamond), and D7 (black up-pointing triangle).
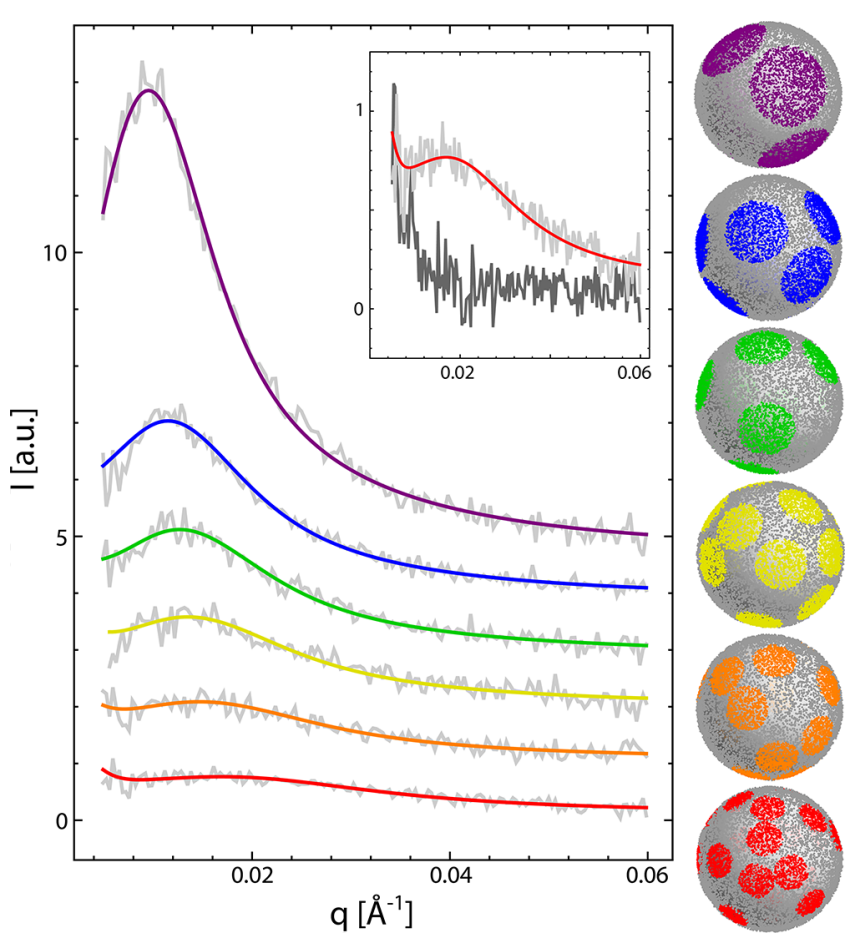

Figure 5. Monte Carlo fits to scattering data at $20{ }^{\circ} \mathrm{C}$. Left, scattering data (light gray lines) and best-fit curves (colored lines) corresponding to compositions D1-D6 (from bottom to top). Inset, data and fit for sample D1 at 20 (light gray line) and $50{ }^{\circ} \mathrm{C}$ (dark gray line) shown on an expanded scale. Right, example Monte Carlo vesicles corresponding to the best-fit parameters at each composition.

curves (Section S2.3, SI). It is likely that for the $\rho=100 \%$ composition, one to a few large domains are present at equilibrium, consistent with observations in GUVs. ${ }^{7,8}$

The observed changes in domain size must be considered in light of those expected due to compositional change alone. With increasing $\rho$, the Lo phase composition shifts to higher fractions of DSPC and Chol (Figure 1), resulting in an increase in the total area of the $\mathrm{Ld}$ (domain) phase. Figure 6 shows the impact of composition on domain size for several values of domain number $N$ (gray lines). While there is an intrinsic increase in domain size with increasing $\rho$, it is small in

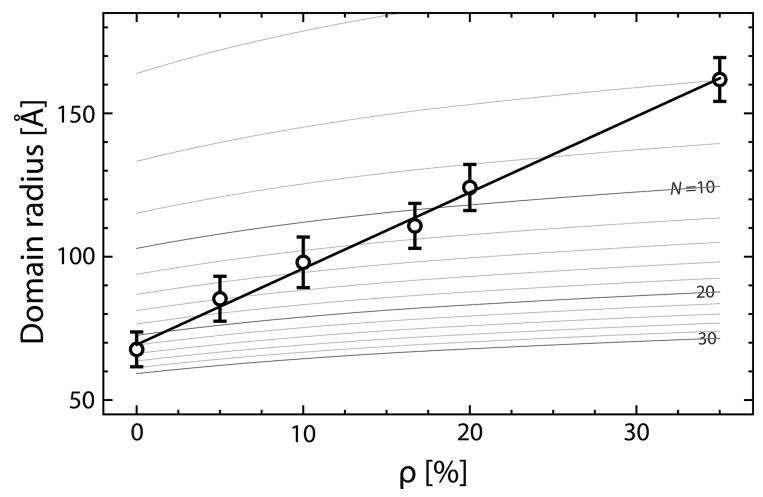

Figure 6. Effective domain sizes at $20{ }^{\circ} \mathrm{C}$ determined from Monte Carlo fits to SANS data. Domain radii reveal a linear increase with the fraction of DOPC for $\rho<35 \%$. Gray lines indicate domain radius corresponding to a fixed number $N$ of domains per vesicle. Error bars $( \pm 2 \sigma)$ were determined by Monte Carlo simulation (SI Section S2.2).

Table 1. Bilayer Thicknesses and Domain Sizes at $20^{\circ} \mathrm{C}$

$\begin{array}{lccccccc} & \mathrm{D} 1 & \mathrm{D} 2 & \mathrm{D} 3 & \mathrm{D} 4 & \mathrm{D} 5 & \mathrm{D} 6 & \mathrm{D} 7 \\ \rho[\%]^{a} & 0 & 5 & 10 & 17 & 20 & 35 & 100 \\ t_{\mathrm{Lo}}(\AA)^{b} & 44.8 & 44.8 & 44.8 & 44.6 & 44.6 & 44.6 & 44.8 \\ t_{\mathrm{Ld}}(\AA)^{b} & 38.4 & 38.0 & 38.0 & 37.6 & 37.7 & 36.7 & 35.1 \\ \Delta t(\AA)^{c} & 6.4 & 6.8 & 6.9 & 7.0 & 6.9 & 7.9 & 9.7 \\ N^{d} & 23 & 16 & 13 & 11 & 9 & 6 & 1-4 \\ R(\AA)^{e} & 68 & 85 & 98 & 111 & 124 & 162 & >225 \\ p^{f} & 4.8 & 4.0 & 3.6 & 3.3 & 3.0 & 2.4 & <2\end{array}$

${ }^{a} \rho \equiv \chi \mathrm{DOPC} /(\chi \mathrm{DOPC}+\chi \mathrm{POPC})[\%] .{ }^{b}$ Lo (Ld) bilayer thickness. ${ }^{c}$ Thickness mismatch. ${ }^{d}$ Number of domains per vesicle. ${ }^{e}$ Domain radius. ${ }^{f}$ Domain perimeter ratio.

comparison to the overall change, and the dominant factor driving the increase in domain size therefore appears to be the reduction of boundary perimeter.

SANS enables a straightforward determination of bilayer thickness, as measured here for the tieline end point compositions shown in Figure 1 and listed in Table S3 (SI). We prepared fully protiated ULVs in $100 \% \mathrm{D}_{2} \mathrm{O}$, contrast conditions that emphasize the total bilayer thickness. Figure 7 (upper panel) shows SANS curves for Ld and Lo compositions corresponding to the coexisting phases at compositions D1 and D7. The local scattering minimum $\left(q_{\min }\right)$ near $0.15 \AA^{-1}$ is related to bilayer thickness. Of the four curves, $q_{\min }$ is smallest and approximately equal for the two Lo compositions (lower curves), indicating a thicker Lo bilayer. Comparing the two Ld compositions (upper curves), $q_{\min }$ shifts from $0.165 \AA^{-1}$ at $\rho=$ 0 to $0.186 \AA^{-1}$ at $\rho=100 \%$, corresponding to a $\sim 4 \AA$ difference in bilayer thickness.

We modeled the data to recover total bilayer thickness as described in Materials and Methods. The Ld and Lo thicknesses corresponding to the coexisting phases at compositions D1- D7 are shown in Figure 7 (lower panel) and listed in Table 1. To within experimental precision, Lo bilayer thickness does not change along the sample trajectory. In contrast, Ld bilayer thickness decreases approximately linearly with increasing $\rho$. Together, these data reveal a thickness mismatch between coexisting phases that increases with increasing DOPC fraction, from $6.4 \AA$ at $\rho=0$ to $9.7 \AA$ at $\rho=100 \%$. These values are comparable to height differences between Ld and Lo phase domains reported for supported sphingomyelin/DOPC/Chol bilayers measured with AFM. ${ }^{11}$ 


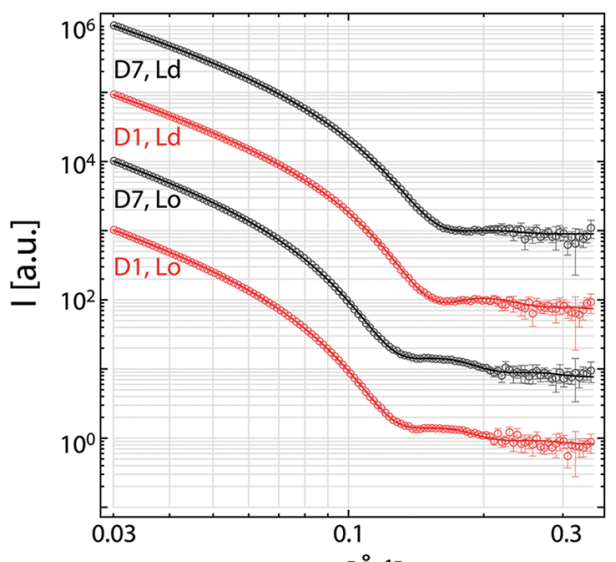

$q\left[\AA^{-1}\right]$

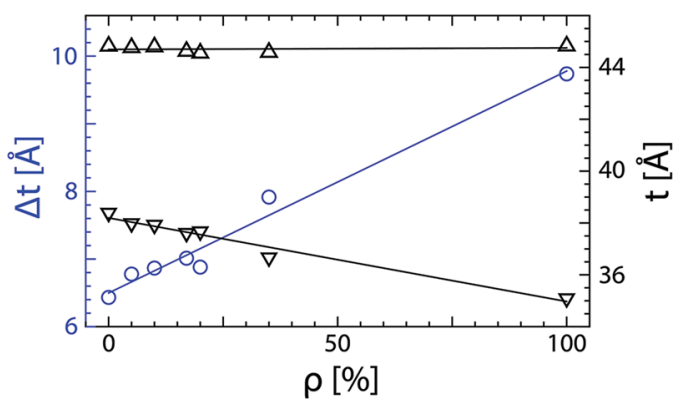

Figure 7. Thickness mismatch between Ld and Lo phases measured by SANS. Upper panel, $20{ }^{\circ} \mathrm{C}$ scattering curves at tieline end point compositions (offset by powers of ten for clarity). Bilayer thicknesses were determined by fits to an asymmetric SLD model (solid lines) and are plotted in the lower panel for Lo (black up-pointing triangle) and Ld (black down-pointing triangle) compositions. The difference in Ld and Lo thickness is also shown at each composition (blue circle). Linear trends are shown with solid lines.

\section{DISCUSSION}

The primary finding of this work is that domain size in $60 \mathrm{~nm}$ ULVs increases continuously and linearly with increasing DOPC fraction in the regime $\rho<35 \%$. Furthermore, the increase cannot be trivially explained by composition-dependent increases in domain area fraction. Rather, it appears to be driven by a reduction in domain perimeter that correlates with the extent of acyl chain unsaturation, consistent with an increased thickness mismatch and energetic cost of domain interface.

A theoretical description of line tension predicts a quadratic dependence on the thickness mismatch between the domain and surround phases. ${ }^{3}$ It is plausible that within the liquid coexistence region of $\mathrm{DSPC} / \mathrm{DOPC} / \mathrm{POPC} / \mathrm{Chol}$, the observed increase in thickness mismatch with increasing acyl chain unsaturation manifests as increased line tension, driving domain coalescence to reduce the boundary energy. In addition to reducing domain perimeter, the bilayer might counteract a thickness mismatch through elastic boundary deformations, and line tension is therefore predicted to also depend on bilayer elastic parameters and curvature. ${ }^{3}$ With literature values for elastic moduli ${ }^{12}$ and lipid intrinsic curvature, ${ }^{13}$ we used existing theory to calculate line tension as a function of mixture composition for our sample trajectory (Section S3, SI). Calculated line tension values fall in the range of $0-5 \mathrm{pN}$, consistent with measurements in similar bilayer mixtures made using micropipet aspiration ${ }^{14}$ and flicker spectroscopy. ${ }^{15,16}$
While the absolute scale depends on particular values of elastic moduli that have not been measured for these compositions, increasing the DOPC fraction likely increases line tension by a few $\mathrm{pN}$, given the bilayer thickness changes observed here.

When similar bilayer compositions were studied using GUVs, optically resolvable domains with spatially modulated features (regularly spaced stripes) appeared suddenly at $\rho=15 \%$, and transitioned to round domains at $\rho=25 \%{ }^{7}$ In contrast, no structure is evident in the $60 \mathrm{~nm}$ ULV SANS data that would suggest a high degree of interdomain organization, nor are distinct scattering or domain size transitions apparent as a function of composition, though the domain size trend is qualitatively similar to that observed in the GUV study. Furthermore, we do not observe complete domain coalescence at $\rho=35 \%$ or $100 \%$, which might be expected from observations of GUVs (though we note that a few domains, rather than a single domain, are often observed in GUVs at compositions considered to exhibit macroscopic phase separation). It is not clear whether these differences are a direct result of differences in curvature or are the result of constraints imposed by the limitation of vesicle size.

Our simple model of round, randomly dispersed domains fits the scattering data well at all compositions examined, but we cannot rule out other domain geometries. Analytical form factors have been derived for a few domain morphologies including annular stripes, but the predicted scattering differences compared to round domains are subtle and occur outside the experimental $q$ window of this study. ${ }^{10}$ It is also possible that the high degree of curvature imposed by small vesicle size may influence domain morphologies and size distributions, ${ }^{17}$ although we note that our finding here of $6.8 \mathrm{~nm}$ domain radius at $\rho=0 \%$ is similar to a FRET-based estimate of $\sim 5 \mathrm{~nm}$ domain radius in micrometer-sized multilamellar vesicles (MLVs) with low curvature. ${ }^{9}$ SANS experiments using larger ULVs could provide important insights into the role of curvature in modulating domain size. Our choice of $60 \mathrm{~nm}$ ULVs was made to avoid contamination with pauci-lamellar vesicles, which cause Bragg scattering due to radial SLD correlations, and are often found in extruded neutral vesicles of $>100 \mathrm{~nm}$ diameter.

Cell membranes are considerably more complex than the model membranes studied here, and we are cautious in extrapolating results from lipid-only model systems to cells. Lingwood and Simons have noted that because the Lo and Ld phase designations in model bilayers are quantitative definitions relating to translational and conformational order, they may not directly map to cell membranes. ${ }^{18}$ However, assuming that cell membrane raft phenomena are described by relatively moreand less-ordered environments, we can speculate as to how cells might utilize lipid composition to control domain size. The present work points to changes in line tension resulting from thickness mismatch as a major determinant of domain size. Whereas DOPC was used in this study to increase the overall level of unsaturation and thin the disordered phase, polyunsaturated lipids may play a similar role in mammalian cells. Other modifications that tend to thin the bilayer, such as short-chain lipids and charged or bulky headgroups, can have the same effect. For example, phosphoglycerol (PG) lipids form bilayers about $3 \AA$ thinner than corresponding PC lipids. ${ }^{19}$ In contrast, the ordered phase thickness may be less variable because it is ultimately constrained by the fully extended lengths of the predominant 16:0 and 18:0 chains and cholesterol. Our study finds that changes in cholesterol 
composition do not measurably alter the thickness of the ordered phase. This observation is consistent with the finding that cholesterol-dependent ordering of saturated PC lipids and bilayer thickening plateau at $\sim 20 \mathrm{~mol} \%$ cholesterol. ${ }^{20}$ All Lo phase compositions within the liquid coexistence region of DSPC/DOPC/POPC/Chol contain $>20 \mathrm{~mol} \%$ cholesterol, and are thus likely to be fully ordered and insensitive to further changes in cholesterol concentration. However, cholesterol may still influence line tension by changing the thickness of the disordered phase, which has a lower baseline cholesterol concentration than the ordered phase. Indeed, micropipet aspiration experiments found a decrease in line tension with increasing cholesterol concentration for coexisting $\mathrm{Ld}$ and Lo phases, ${ }^{14}$ though bilayer thickness was not measured. In vivo, proteins are also believed to be important determinants of bilayer thickness. $^{21}$

We have shown that changes in domain size are strongly correlated with bilayer thickness mismatch, implying that line tension is a primary driving force for domain coalescence. If line tension were the only relevant energetic consideration, then in principle, any system with coexisting liquid domains would achieve an equilibrium state of a single, round domain. ${ }^{22} \mathrm{~A}$ major unanswered question is what competing energetic term is responsible for the breakup of domains under conditions of low line tension. Several potential mechanisms have been proposed, including an entropic penalty for domain merger, ${ }^{4}$ long-range domain repulsion deriving from lipid dipole moment, ${ }^{23}$ or curvature. $^{24-27}$ Such mechanisms depend on differential properties of coexisting phases, and definitive answers are now within reach as the considerable effort put toward solving multicomponent phase diagrams bears fruit.

\section{CONCLUSIONS}

SANS reliably detects the formation of nanoscopic domains in $60 \mathrm{~nm}$ ULVs. With regard to the phase behavior of DSPC/ DOPC/POPC/Chol, observations of phase separation with SANS are in agreement with FRET and ESR measurements of MLVs. ${ }^{9}$ Among these techniques, neutron scattering is unique in that perturbations to membrane composition are minimal, requiring only deuteration of some lipid components, rather than the addition of extrinsic probes. This advantage may be of great value for systems that are particularly sensitive to artifactual domain formation due to the presence of fluorophores. $^{28}$

With the ratio of saturated lipid and cholesterol held constant, the size of membrane domains increases with the fraction of unsaturated acyl chains in DSPC/DOPC/POPC/ Chol. The increase in domain size correlates with a linear increase in the thickness difference between the coexisting phases. In particular, Ld bilayer thickness decreases as the DOPC fraction increases, while changes in Lo bilayer thickness are negligible. We conclude that lipid remodeling strategies that alter the thickness of the Ld phase are a plausible mechanism for controlling raft size in cells of homeothermic organisms.

Together, the observation of increasing domain size with increasing thickness difference lends support to theories relating line tension to hydrophobic mismatch of coexisting phases. To our knowledge, this is the first direct evidence from free-floating bilayers supporting these theories.

\section{MATERIALS AND METHODS}

DSPC, DSPC- $\mathrm{d}_{70}$, DOPC, and POPC were purchased as lyophilized powders from Avanti Polar Lipids (Alabaster, AL). Cholesterol was purchased from $\mathrm{Nu}$ Chek Prep (Elysian, MN). Stock solutions were prepared by dissolving lipid or cholesterol in HPLC grade chloroform. Phospholipid purity of $>99 \%$ was confirmed by thin-layer chromotography (TLC) on washed, activated Adsorbosil TLC plates (Alltech, Deerfield, IL), developed with chloroform/methanol/water in a 65/ $25 / 4$ ratio. Concentration of phospholipid stocks was determined to within $1 \%$ by inorganic phosphate assay. Ultrapure $\mathrm{H}_{2} \mathrm{O}$ was obtained from a Barnstead purification system (Dubuque, IA), and 99.8\% $\mathrm{D}_{2} \mathrm{O}$ was purchased from Alfa Aesar (Ward Hill, MA).

Sample Preparation. Unilamellar vesicles (ULVs) were prepared by extrusion as follows. Lipid mixtures were prepared by transferring desired volumes of chloroform stock solutions to a glass culture tube with a syringe (Hamilton USA, Reno, NV). Chloroform was removed with an $\mathrm{N}_{2}$ stream and gentle heating, followed by drying in vacuo for a minimum of $12 \mathrm{~h}$. Dry lipid films were hydrated with an appropriate $\mathrm{D}_{2} \mathrm{O} / \mathrm{H}_{2} \mathrm{O}$ mixture preheated to $50{ }^{\circ} \mathrm{C}$, followed by vigorous vortexing to disperse the lipid. The resulting multilamellar vesicle (MLV) suspension was incubated at $50{ }^{\circ} \mathrm{C}$ for $1 \mathrm{~h}$, and then subjected to 5 freeze/thaw cycles between -80 and $50{ }^{\circ} \mathrm{C}$ to reduce the average number of lamellae and facilitate extrusion. ULVs were prepared with a hand-held miniextruder (Avanti Polar Lipids, Alabaster, AL), assembled with a $50 \mathrm{~nm}$ pore-diameter polycarbonate filter and heated to $50{ }^{\circ} \mathrm{C}$. The suspension was passed through the filter a minimum of 31 times, in all cases using an odd number of passes to minimize contamination with unextruded starting material. ULVs were measured within $24 \mathrm{~h}$ of extrusion. Final sample concentrations were $10-15 \mathrm{mg} / \mathrm{mL}$, which allows for sufficient water between vesicles to eliminate the interparticle structure factor, thereby simplifying data analysis. $^{29}$

SANS Measurements. Neutron scattering experiments were performed at the CG-3 Bio-SANS instrument of the High Flux Isotope Reactor (HFIR), and at the BL-6 extended Q-range smallangle neutron scattering (EQ-SANS) instrument of the Spallation Neutron Source (SNS); both instruments are located at Oak Ridge National Laboratory (ORNL). ULV suspensions were loaded into 1 mm path-length quartz banjo cells (Hellma USA, Plainview, NY) and mounted in a temperature-controlled cell holder with $\sim 1{ }^{\circ} \mathrm{C}$ accuracy. Bio-SANS data were taken at sample-to-detector distances (SDD) of 1.7 and $14.5 \mathrm{~m}$, using $6 \AA$ wavelength neutrons (fwhm 15\%), resulting in a total scattering vector of $0.005<q<0.3 \AA^{-1}$. EQ-SANS data were taken at a $2.0 \mathrm{~m}$ SDD with a $2.5-5.5 \AA$ wavelength band for a total scattering vector of $0.01<q<0.3 \AA^{-1}$. Scattered neutrons were collected with a two-dimensional $(1 \mathrm{~m} \times 1 \mathrm{~m}){ }^{3} \mathrm{He}$ position-sensitive detector (ORDELA, Inc., Oak Ridge, TN) with $192 \times 192$ pixels (BioSANS) or $256 \times 192$ pixels (EQ-SANS). The $2 \mathrm{D}$ data were reduced using software provided by ORNL. During reduction, data were corrected for detector pixel sensitivity, dark current, and sample transmission. Background scattering from water was subtracted, and the one-dimensional scattering intensity $I$ vs $q[q=4 \pi \sin (\theta) / \lambda$, where $\lambda$ is the neutron wavelength and $2 \theta$ is the scattering angle relative to the incident beam] was obtained by radial averaging of the corrected $2 \mathrm{D}$ data.

Monte Carlo Modeling. SANS profiles were modeled with a modified coarse-graining method, ${ }^{30}$ full details of which are provided as SI (Section S2). Vesicles were approximated as spherical shells of radius $R$ and thickness $t$ corresponding to the hydrophobic thickness of the bilayer, with polydispersity assumed to follow a Schulz distribution. The shell volume was further divided into one or more randomly placed, nonoverlapping caps (domains). For the compositions studied, these domains are considered to be liquid-disordered phase dispersed in a continuous liquid-ordered background. Random points were generated within the shell volume in proportion to the SLD contrast of the phases using a rejection algorithm (i.e., points were uniformly generated within the shell and tested for inclusion in a domain until both the domain and surrounding volumes accumulated the desired number of points). The pair distance distribution $P(r)$ for the vesicle was then calculated from the set of random points following Henderson, ${ }^{31,31}$ and the procedure was repeated for $10^{5}$ vesicles to obtain an ensemble average. 
Bilayer Thickness Determination. SANS intensity for protiated bilayers in $100 \% \mathrm{D}_{2} \mathrm{O}$ was modeled by inverse Fourier methods, using an asymmetric SLD profile to account for different average structure of the inner and outer leaflets (Section S4, SI). The total bilayer thickness was taken to be the distance between the half-height SLD of the linearly increasing portions of the SLD profile. The thickness determined in this fashion is related to the distance between the interfacial regions (i.e., lipid/water boundaries) of the inner and outer leaflets. While the distance determined by this simple model is not rigorously connected to the steric bilayer thickness, observed trends are expected to be a reliable representation of thickness differences for similar bilayer compositions such as those studied here.

\section{ASSOCIATED CONTENT}

\section{S Supporting Information}

Three stand-alone tables, and four sections containing four figures and ten equations. This material is available free of charge via the Internet at http://pubs.acs.org.

\section{AUTHOR INFORMATION}

\section{Corresponding Author}

heberlefa@ornl.gov; katsarasj@ornl.gov

\section{Notes}

The authors declare no competing financial interest.

\section{ACKNOWLEDGMENTS}

Support was received from the Laboratory Directed Research and Development Program of Oak Ridge National Laboratory (to J.K. and R.F.S.), managed by UT-Battelle, LLC, for the U.S. Department of Energy (DOE), and from National Science Foundation research award MCB 0842839 (to G.W.F.). This work acknowledges additional support from the DOE Office of Biological and Environmental Research, for the BioSANS instrument at the ORNL Center for Structural Molecular Biology, and from the Scientific User Facilities Division of the DOE Office of Basic Energy Sciences, for the EQ-SANS instrument at the ORNL Spallation Neutron Source. These facilities are managed for DOE by UT-Battelle, LLC under contract no. DE-AC05-00OR2275. A portion of this research was conducted using the resources of the Cornell Center for Advanced Computing, which receives funding from Cornell University, the National Science Foundation, and other leading public agencies, foundations, and corporations. We thank Renee Manning for providing the cover artwork.

\section{REFERENCES}

(1) Lingwood, D.; Simons, K. Science 2010, 327, 46.

(2) Elson, E. L.; Fried, E.; Dolbow, J. E.; Genin, G. M. Ann. Rev. Biophys. 2010, 39, 207.

(3) Kuzmin, P. I.; Akimov, S. A.; Chizmadzhev, Y. A.; Zimmerberg, J.; Cohen, F. S. Biophys. J. 2005, 88, 1120.

(4) Frolov, V. A. J.; Chizmadzhev, Y. A.; Cohen, F. S.; Zimmerberg, J. Biophys. J. 2006, 91, 189.

(5) Marsh, D. Biochim. Biophys. Acta 2009, 1788, 2114.

(6) Feigenson, G. W. Biochim. Biophys. Acta 2009, 1788, 47.

(7) Konyakhina, T. M.; Goh, S. L.; Amazon, J.; Heberle, F. A.; Wu, J.; Feigenson, G. W. Biophys. J. 2011, 101, L8.

(8) Zhao, J.; Wu, J.; Heberle, F. A.; Mills, T. T.; Klawitter, P.; Huang, G.; Costanza, G.; Feigenson, G. W. Biochim. Biophys. Acta 2007, 1768, 2764.

(9) Heberle, F. A.; Wu, J.; Goh, S. L.; Petruzielo, R. S.; Feigenson, G. W. Biophys. J. 2010, 99, 3309.

(10) Anghel, V. N. P.; Kučerka, N.; Pencer, J.; Katsaras, J. J. Appl. Crystallogr. 2007, 40, 513.
(11) Garcia-Saez, A. J.; Chiantia, S.; Schwille, P. J. Biol. Chem. 2007, $282,33537$.

(12) Semrau, S.; Idema, T.; Holtzer, L.; Schmidt, T.; Storm, C. Phys. Rev. Lett. 2008, 100, 088101.

(13) Boulgaropoulos, B.; Rappolt, M.; Sartori, B.; Amenitsch, H.; Pabst, G. Biophys. J. 2012, 102, 2031.

(14) Tian, A. W.; Johnson, C.; Wang, W.; Baumgart, T. Phys. Rev. Lett. 2007, 98, 208102.

(15) Esposito, C.; Tian, A.; Melamed, S.; Johnson, C.; Tee, S. Y.; Baumgart, T. Biophys. J. 2007, 93, 3169.

(16) Honerkamp-Smith, A. R.; Cicuta, P.; Collins, M. D.; Veatch, S. L.; den Nijs, M.; Schick, M.; Keller, S. L. Biophys. J. 2008, 95, 236.

(17) Pencer, J.; Jackson, A.; Kučerka, N.; Nieh, M. P.; Katsaras, J. Eur. Biophys. J. 2008, 37, 665.

(18) Lingwood, D.; Ries, J.; Schwille, P.; Simons, K. Proc. Natl. Acad. Sci. U.S.A 2008, 105, 10005

(19) Pan, J. J.; Heberle, F. A.; Tristram-Nagle, S.; Szymanski, M.; Koepfinger, M.; Katsaras, J.; Kučerka, N. Biochim. Biophys. Acta 2012, 1818, 2135.

(20) Pan, J.; Mills, T. T.; Tristram-Nagle, S.; Nagle, J. F. Phys. Rev. Lett. 2008, 100, 198103.

(21) Mitra, K.; Ubarretxena-Belandia, I.; Taguchi, T.; Warren, G.; Engelman, D. M. Proc. Natl. Acad. Sci. U.S.A 2004, 101, 4083.

(22) Akimov, S. A.; Kuzmin, P. I.; Zimmerberg, J.; Cohen, F. S. Phys. Rev. E 2007, 75, 011919.

(23) Travesset, A. J. Chem. Phys. 2006, 125, 084905

(24) Hu, J. L.; Weikl, T. R.; Lipowsky, R. Soft Matter 2011, 7, 6092.

(25) Ursell, T. S.; Klug, W. S.; Phillips, R. Proc. Natl. Acad. Sci. U.S.A. 2009, 106, 13301

(26) Semrau, S.; Idema, T.; Schmidt, T.; Storm, C. Biophys. J. 2009, 96, 4906.

(27) Rozovsky, S.; Kaizuka, Y.; Groves, J. T. J. Am. Chem. Soc. 2005, $127,36$.

(28) Zhao, J.; Wu, J.; Shao, H. L.; Kong, F.; Jain, N.; Hunt, G.; Feigenson, G. Biochim. Biophys. Acta 2007, 1768, 2777.

(29) Kučerka, N.; Pencer, J.; Sachs, J. N.; Nagle, J. F.; Katsaras, J. Langmuir 2007, 23, 1292.

(30) Pencer, J.; Mills, T.; Anghel, V.; Krueger, S.; Epand, R. M.; Katsaras, J. Eur. Phys. J. E 2005, 18, 447.

(31) Henderson, S. J. Biophys. J. 1996, 70, 1618. 